\title{
MOLECULAR REACTION KINETICS INSIDE CHANNEL PORES: DELAYED FLUORESCENCE OF NAPHTHALENE IN METHANOL
}

\author{
Jagdish PRASAD and Raoul KOPELMAN \\ Department of Chemistry, The University of Michigan, Ann Arbor, MI 48109-1055, USA
}

Received 14 February 1989 ; in final form 8 March 1989

\begin{abstract}
A one-dimensional, diffusion-controlled, reaction is followed in time by phosphorescence (monitoring reactant) and delayed fluorescence (monitoring product). The triplet (excited) naphthalene molecules move in a methanol solution, confined inside the cylindrical channels of a channel-pore polycarbonate membrane. The reaction product is a transient photodimer or excimer, giving delayed fluorescence. In large radius $(1 \mu \mathrm{m})$ channel-pore membranes, the reaction kinetics are classical, with a heterogeneity exponent $h=0$. However, in the small radius $(0.025 \mu \mathrm{m})$ channel-pore membranes the kinetics approach that of a onedimensional environment, $h=1 / 2$. For membranes with intermediate channel radius $(0.1 \mu \mathrm{m})$, the reaction exhibits a "crossover" behavior which can be approximated via "fractal-like" kinetics.
\end{abstract}

Many chemical and biological processes occur in pores or micro-channels. Thus it is of practical interest to study molecular reactions inside microchannels. The molecular kinetics inside channels may be very different from the conventional kinetics, depending on the geomelry and size of the channels. In conventional chemical kinetics, the order of an elementary reaction is equal to its molecularity, and thus given by a natural number $(1,2,3 \ldots)$. Recently, it has been demonstrated both theoretically and experimentally that the reaction order may differ significantly from its molecularity [1-10]. In particular, this may be true for reactions inside random networks of micro-channels $[8,9]$. In this work, we demonstrate that the reaction order, in fact, depends on the size of the channels and that a "fractal-like" kinetics model works well for this type of systems.

Here we report the transient photodimerization of naphthalene $(\mathrm{N})$ in cylindrical micro-channels of various diameters. Transient photodimerization of naphthalene has been studied in detail [11]:

$\mathrm{N}^{+}+\mathrm{N}^{+} \rightarrow \mathrm{N}^{+}+\mathrm{N} \rightarrow \mathrm{N}+\mathrm{N}+h \nu$,

Here $\mathrm{N}$ is a naphthalene molecule in its first triplet state, $\mathrm{N}^{+}+\mathrm{N}$ is a transient singlet "excimer", $\mathrm{N}^{+}$is a naphthalene moleculc in its first excited singlet state and $h \nu$ is the ultraviolet fluorescence quantum. The first step of eq. (1) is rate determining and diffusion controlled. It is clearly an elementary photochemical (photophysical) reaction. It is also quite analogous to charge exchange reactions which may occur in biological membranes $\left(A^{+}+A^{+} \rightarrow A^{2+}+A\right.$, etc. $)$. We notice that the reactant $\left(\mathrm{N}^{+}\right)$is produced at will via direct triplet excitation. At a given light level a steady state is produced. Both the steady state and the relaxation from it are monitored. The instantaneous reactant concentration is monitored (in time) via the green phosphorescence (the "natural decay" of the first excited triplet). The instantaneous product concentration is monitored via the ultraviolet delayed fluorescence. In the relaxation part of the experiment the product's instantaneous concentration is directly proportional to the reaction rate. We thus have a highly controlled and doubly time monitored photoreaction.

In the "fractal kinetics" formalism the dimerization rate is given by

$$
-\mathrm{d} C / \mathrm{d} t=k t^{-h} C^{2}
$$

where $C$ is the monomer concentration, $t$ the time, $k$ a constant and $h$ the heterogeneity exponent. For homogeneous three-dimensional reactions (2), $h=0$ and $k$ is the rate constant. For one-dimensional reactions, $h=1 / 2$. For reactions in fractal domains [3], 
$0<h<1$. Furthermore, $h=1-\frac{1}{2} d_{s}$, where $d_{s}$ is the effective spectral dimension $[3,12]$, which, for fractals, is in the range $0<d_{\mathrm{s}}<2$. We thus call a reaction "fractal-like" if $h>0$ (including the special one-dimensional case where $d_{\mathrm{s}}=1$ and $h=1 / 2$ ). We note that eq. (2) is equivalent [8] to a fractal reaction order $X=(h-2) /(h-1)$.

In reality, we have often found before [9-11] that the excited naphthalene species exists in two forms: mobile species and trapped species. If trapped species are involved, a pseudo-first-order reaction may result. The steps involved are as follows: (1) The mobile excitation is trapped out by the lower energy lcvels of the "trap". This process depletes some of the mobile excitations. (2) A fusion process occurs: this is mostly "heterofusion" between a free and a trapped excitation. (3) The heterofusion further depletes the free excitation, but the trapped excitation density is nearly constant in time, $T+T^{\prime} \rightarrow T^{\prime}$, because most fusion products are again triplet traps. The reason for this is that the trap or aggregate excitations are red-shifted compared to the naphthalene monomers; for a more complete discussion see refs. [13-15]. Thus we rewrite eq. (2) as

$-\mathrm{d} C / \mathrm{d} t=K t^{-h} C C^{\prime}=K^{\prime} t^{-h} C$.

Here $C^{\prime}$ is the nearly constant density of the trap excitation, $K^{\prime}=K C^{\prime}$, and $\left(2^{\prime}\right)$ is a pseudo-first-order reaction. As usual, the delayed fluorescence is proportional to the fusion rate, $F_{\varnothing C}|\mathrm{~d} C / \mathrm{d} t|$. The observed phosphorescence, after corrections for the baseline at longer times, is $P(t) \propto C(t)$. Thus

$F=\bar{K}^{-h} P$

and

$\log (F / P)=-h \log (t)+\log (\bar{K})$.

Data analysis is done according to eq. ( $\left.3^{\prime}\right)$ : plotting $\log (F / P)$ versus $\log (t)$ which gives a slope of $-h$ (table 1).

The optical setup and sample preparation have been described earlier $[9,10]$. The only significant change involves the use of channel-pore (Nuclepore) polycarbonate membranes [11] instead of ordinary (random) porous membranes. These membranes come with cylindrical channels (fig. 1). While a given membranc has channels of uniform diameter, membranes with different channel diameters are
Table 1

Heterogeneity exponents for naphthalene-ethanol solution in polycarbonate channel-pore membranes (200 K)

\begin{tabular}{|c|c|}
\hline 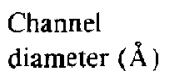 & $h^{\mathbf{a})}$ \\
\hline 20000 & 0.07 \\
\hline \multirow[t]{2}{*}{$2000^{b)}$} & 0.06 (st) \\
\hline & 0.48 (lt) \\
\hline 500 & 0.41 \\
\hline
\end{tabular}

a) Exponent. Error bars are \pm 0.02 .

b) Cross-over regime. Two values correspond to two regimes: short times (st) and long times (1t).

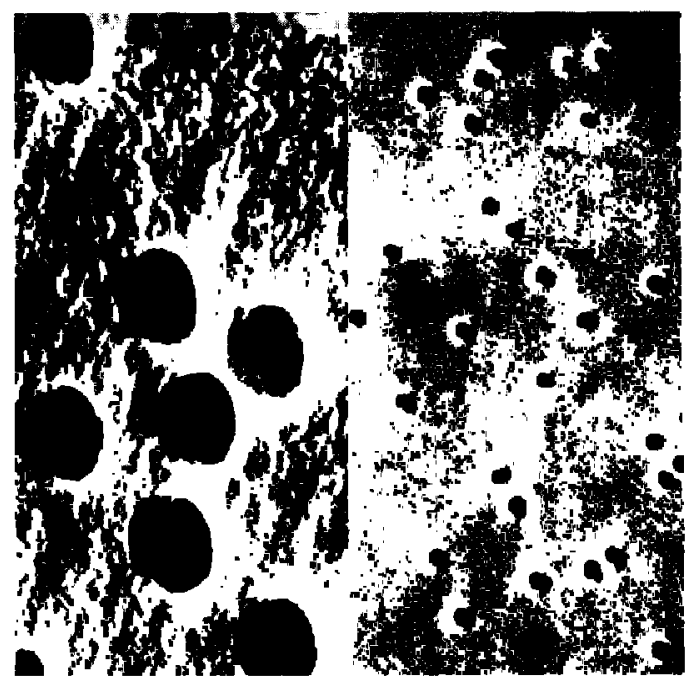

Fig. 1. Polycarbonate channel-pore membranes (Nuclepore, Pleasanton, California). Enlargement about $\times 10^{4}$. Channel length is $6 \mu \mathrm{m}$ and the radius of the channels on the left is $1 \mu \mathrm{m}$ while that on the right is $0.1 \mu \mathrm{m}$.

available and we used them in the range of 500 to $20000 \AA$. We note in addition that the membranes are optically transparent and scatter little light when in solution.

We assume that the mobile excitation species are the diffusing naphthalene molecules (excited "monomers") while the trapped species are an excited naphthalene aggregate or a monomer adsorbed at the channel wall. While the fraction of trapped species is small, the fraction of excited trapped species is large, due to excitation supertrapping [14]. Both aggregate and adsorbed monomer have lower excited state levels than the free monomer, giving 


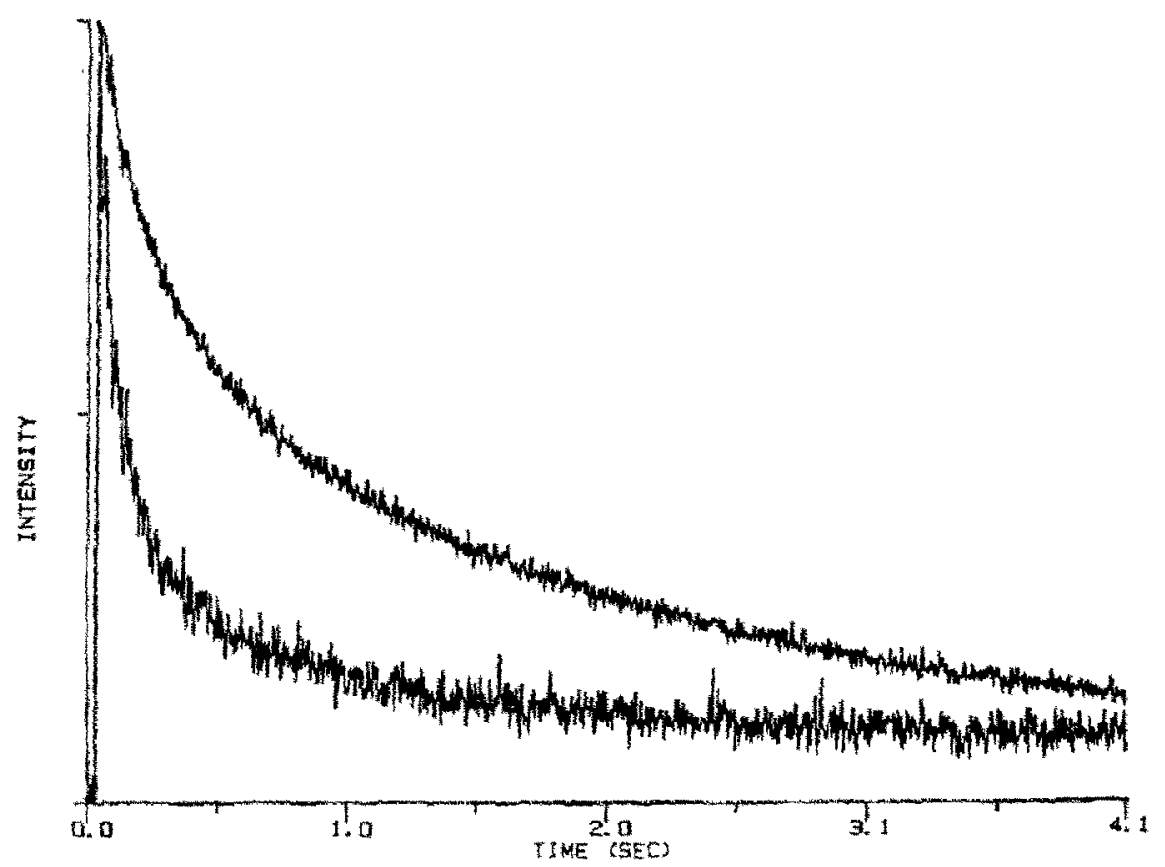

Fig. 2. Phosphorescence (top) and delayed funtescence (bottom) decays of naphthalene dopect membrane with channel diameters of $500 \AA$ at $200 \mathrm{~K}$. Intensity of two decay curves is different.

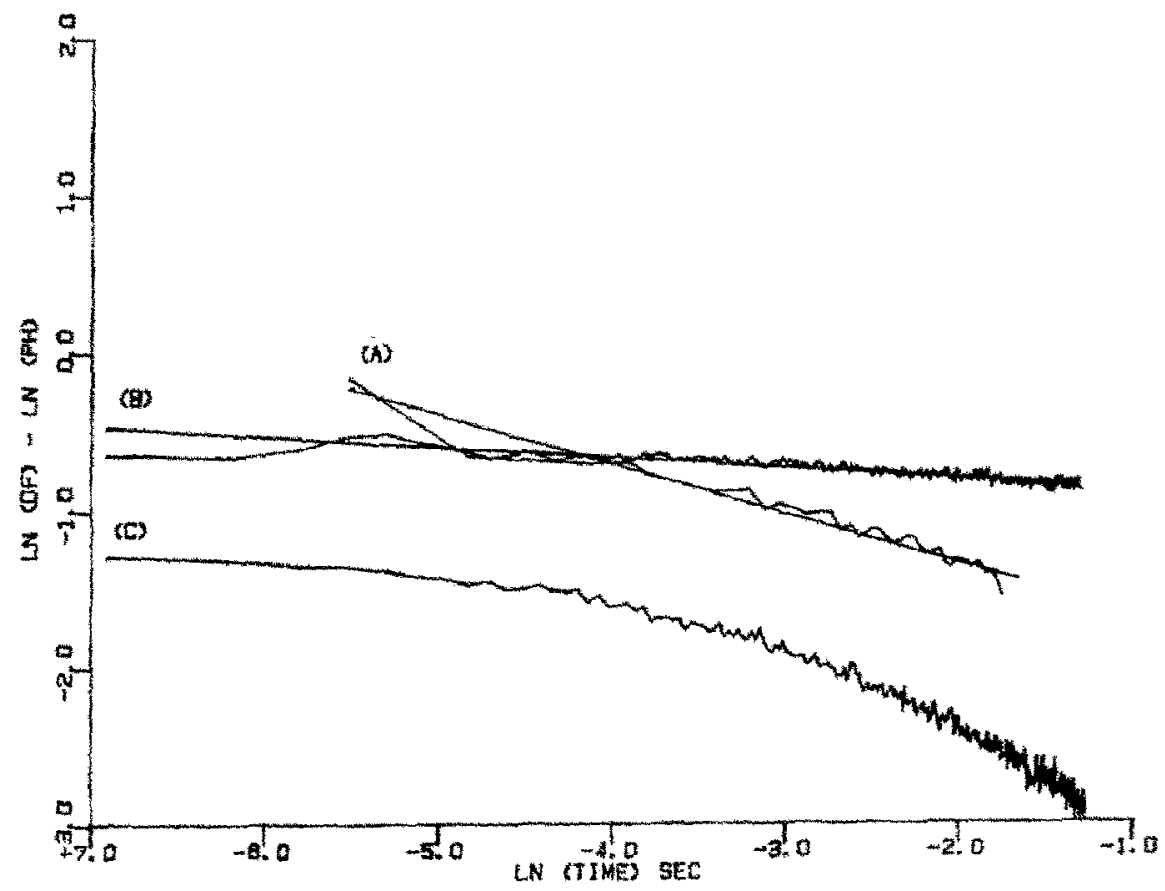

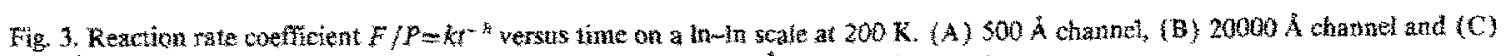
2000 A channetiameters. Nute the uross-over behavior of the 2000 A chanusls (see text). 
$T \rightarrow T^{\prime}$ and $T+T^{\prime} \rightarrow T^{\prime}$. This is the condition that justifies eqs. $\left(2^{\prime}\right)$ and (3). Data are shown in figs. 2 and 3 . These data are consistent with our "heterofusion" model for all $h$ values but are inconsistent with the "homofusion" model of eq. (2). We note that this model of heterofusion explains previous porous membrane experiments as well as experiments on polymer blends, molecularly doped polymers and thin crystalline film samples [9-15]. For homogeneous heterofusion kinetics (the classical behavior) one expects horizontal lines, giving $h=0$.

We observe (table 1) that the $20000 \AA$ channels yield a value $h=0.07 \pm 0.02$ while $500 \AA$ channels yield a value $h=0.41 \pm 0.02$. These two limiting values are in good agreement with the theoretically expected values of $h=0$ and $h=1 / 2$, for three-dimensional and one-dimensional models, respectively. A crossover (between $h=0$ and $h=1 / 2$, fig. 3 ) behavior (1) occurs at diameters of about $2000 \AA$. This gives an upper limit on the diffusion length of the molecules in solution. Obviously, for channels with a diameter of $20000 \AA$, the molecules hardly feel the channel boundaries, while in channels with a diameter of only $500 \AA$, the molecules are severely confined.

In summary, (1) we have been able to observe the photodimerization of naphthalene inside cylindrical channels of various diameters; (2) the overall diffusion length is estimated to be on the order of 2000 $\AA$ at $200 \mathrm{~K} ;$ (3) in narrow channels molecular diffusion is one dimensional in nature while in wide channels it is essentially classical, that is, homogeneous three dimensional; (4) the fractal-like kinetics model works well in low-dimensional nonfractal systems.
This research was partially supported by NSF Grant DMR 8801120. Acknowledgement is also made to the Donors of The Petroleum Fund, administered by The American Chemical Society for their partial support.

\section{References}

[1] C.L. Yang, P. Eyesque and M.A. El-Sayed, J. Phys. Chem. 89 (1985) 3442 .

[2] P. Evesque and J. Duran, J. Chem. Phys. 82 (1984) 3198.

[3] P.W. Klymko and R. Kopelman, J. Luminescence 24/25 (1982) 456: J. Phys. Chem. 86 (1982) 3686; 87 (1983) 4565.

[4] R. Kopelman, J. Hoshen, J.S. Newhouse and P. Argyrakis, J. Stat. Phys. 30 (1983) 355

[5] R. Kopclman, P.W. Klymko, J.S. Newhouse and L.W. Anacker, Phys. Rev. B 29 (1984) 3747.

[6] G. Zumofen, A. Blumen and J. Klafter, J. Chem. Phys. 82 (1985) 3198.

[7] L.W. Anacker and R. Kopelman, J. Chem. Phys. 81 (1984) 6402.

[8] L.W. Anacker, R.P. Parson and R. Kopelman, J. Phys. Chem. 89 (1985) 4758.

[9] R. Kopelman, S. Parus and J. Prasad, Phys. Rev. Letters 56 (1986) 1742.

[10] J. Prasad and R. Kopelman, J. Phys. Chem. 91 (1987) 265.

[11] J. Prasad and R. Kopelman, Phys. Rev. Letters 59 (1987) 2103.

[12] E. Doller and Th. Förster, Z. Physik. Chem. 31 (1962) 274.

[13] R. Kopelman, J. Stat. Phys. 42 (1986) 185.

[14] T. Gentry and R. Kopelman, J. Chem. Phys. 81 (1984) 3014, 3022.

[15] R. Kopelman, Science $241(1988) 1620$. 